\title{
Proceeding
}

8th INSHS International Christmas Sport Scientific Conference, 5-7 December 2013. International Network of Sport and Health

Science. Szombathely, Hungary

\section{The analysis of individual tactics in the women's water polo}

\author{
SALVATORE NAPOLITANO , DANIELA TURSI \\ Department Physical Education and Wellness, University of Naples "Parthenope", Naples, Italy
}

\begin{abstract}
Napolitano, S. \& Tursi, D. (2014). The analysis of individual tactics in the women's water polo. J. Hum. Sport Exerc., 9(Proc1), pp.S576-S583. In water polo lacks a codified methodology for tactics training, which is thus only left to coach's discretion. The aim of this study is to create a methodology that is to fill such vacancy. Aim of the work is to verify the efficacy of different attack patterns, in order to create a codified methodology for teaching water polo through tactics. The data, collected via video-analysis, were analyzed trough which returned basic descriptive statistics and the correlation coefficient of each pattern with events outcomes. The research approach is integrated and consists of 3 distinct methods: case study (9 matches of the Italian Serie A1 Women's Championship, for the analysis of matches, action research method for coach contribution, and theoretical-argumentative method to deduce a theoretical framework in which define the data processing. The data, collected via Dartfish TeamPro, for each athlete were established 4 patterns, we have provided quantitative data, we also have processed data from the analysis (qualitative) of the video. An evaluation table was constructed by combining, for each single event, the Boolean evaluation of the coach on the compliance of patterns with the event final outcome. Then were processed, Confrontations of pattern design with Dartfish screenshot of pattern implemented during game; Basic descriptive statistics. Linear regression scatter plot for single patterns; the survey of data is entrusted to performance analysis, carried out with the help of a water polo coach, a statistician and a performance analyst. The assessment of compliance for the tactical patterns is entrusted to the coach, on the basis of the video analysis-aided confrontation, of 4 patterns previously identified for each athlete in the relative phases of the game against pattern effectively implemented during match. A total of 186 Plate were analyzed, about 20 Plate every athlete. The results showed a general efficacy of tactical patterns, but showed significant differences within correlation coefficients of single patterns. A more consistent data base is needed, in order to establish direct, evident and general relationship between so calculated coefficient and pattern efficacy, and the research team is conscious of internal validity of this kind of qualitative analysis, which can't extend, without adjustments, to other teams. Although, analysis results represents a tool for the coach, in order to better train team in next season, which were an aim of action research, and showed a general trend on tactical pattern efficacy, which will be deeply investigated in future works. Key words: VIDEOANALYSIS, WATER POLO TACTIS, PERFORMANCES.
\end{abstract}

Corresponding author. Department Physical Education and Wellness, University of Naples "Parthenope", Naples, Italy. E-mail: daniela.tursi1@gmail.com 8th INSHS International Christmas Sport Scientific Conference, 5-7 December 2013. International Network of Sport and Health Science. Szombathely, Hungary.

JOURNAL OF HUMAN SPORT \& EXERCISE ISSN 1988-5202

(c) Faculty of Education. University of Alicante

doi:10.14198/jhse.2014.9.Proc1.49 


\section{INTRODUCTION}

This pilot work represents an attempt to develop methods and consequential tools to analyze, and then train, tactical water polo side, knowing that

"the coaches of team sports analyze matches and performances of team and opposing teams to get useful data in coaching" (Hughes \& Franks, 2008) and that,

"currently, the process of training, its organization, and teaching methodology need more knowledge on the qualitative aspects of sports performance (Schmidt \& Wrisberg 2008)".

Nine women water polo matches, during season 2011/2012 (Italian Serie A1), have been analyzed by a water polo coach, helped by a statistician and a performance analyst.

Purpose of the analysis process was

to identify single events during the matches,

to examine the tactical patterns implemented in these events,

to obtain by the coach an evaluation on tactical pattern compliance and then

to put this compliance in relation to event's outcome.

Aim of the work is to verify the efficacy of different attack patterns, when they were well-performed, in order to create a codified methodology for teaching water polo through tactics. The data, collected via Dartfish TeamPro Software, were analyzed trough "Water polo Tactics Analyzer" software, developed as a webbased application at University of Salerno and released under GPL license, which returned basic descriptive statistics and the correlation coefficient of each pattern with events outcomes.

The results show a positive and statistically significant correlation coefficient between tactical compliance and events outcome, and highlight the need for developing a common methodology for teaching water polo through tactics, confirming once again that

"the practical value of performance analysis is that well-chosen performance indicators highlight good and bad techniques or team performances (Hughes, 2004)".

\section{METHODS}

The research approach is integrated and consists of 3 distinct methods:

case study (9 matches of the Italian Serie A1 Women's Championship, season 2011/2012, played by the Volturno s.c.) for the analysis of matches,

action research method for coach contribution,

and theoretical-argumentative method to deduce a theoretical framework in which define the data processing.

\section{Participants}

Team Volturno S.C. (9 matches of the Italian Serie A1 Women's Championship, season 2011/2012).

\section{Procedures}

The survey of data is entrusted to performance analysis, carried out with the help of a water polo coach, a statistician and a performance analyst.

The assessment of compliance for the tactical patterns is entrusted to the coach, on the basis of the video analysis-aided confrontation of attack pattern design against attack pattern effectively implemented during 
match. The research team examined matches with Dartfish Team Pro, isolating single key Plate relative to attack events, identifying the implemented attack pattern, then the coach expressed an evaluation on attack pattern compliance.

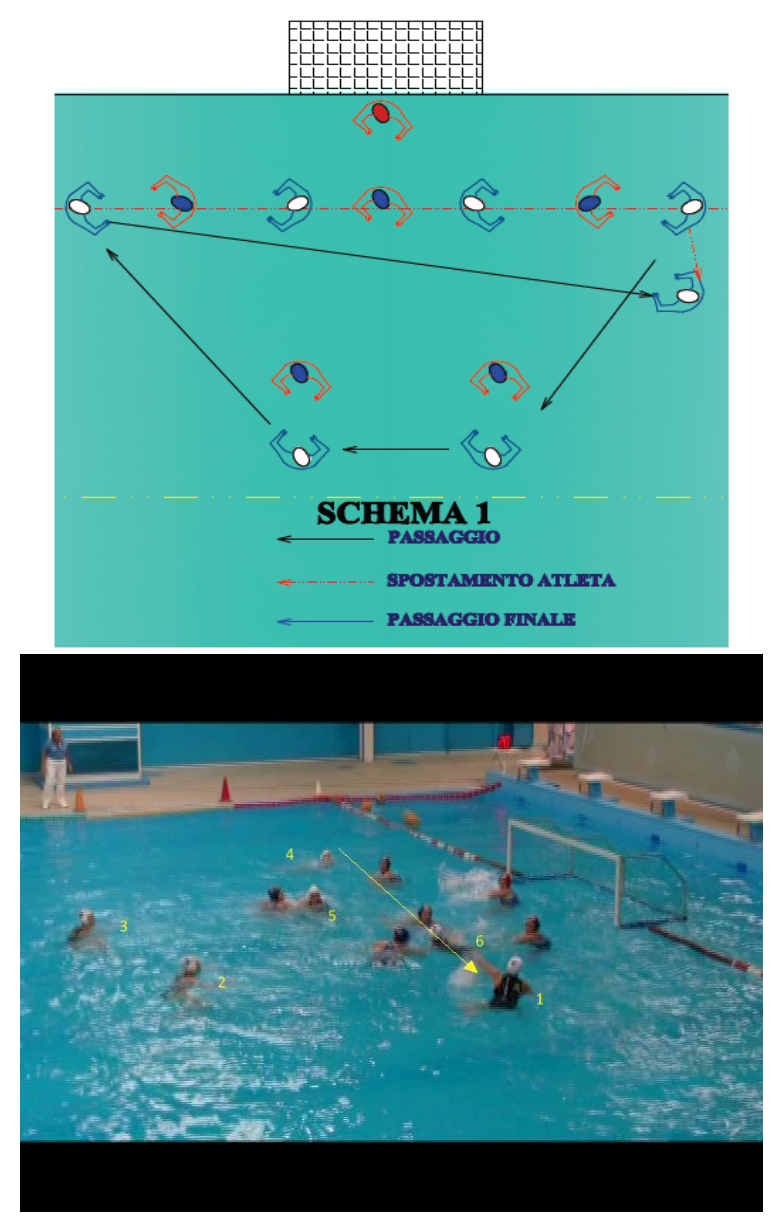

Figure 1 - Plate 1. Confrontation between pattern design and effectively implemented pattern during match for attack pattern named "schema1"

A spreadsheet containing, for each row, the match id, the event id, the attack pattern id, the coach's evaluation (compliant/non compliant) and the event outcome (goal / non goal) was filled. This data sheet is processed through the "water polo Tactics analyzer software", which produces basic descriptive statistics and the correlation coefficient of each well-implemented attack pattern with events outcomes.

In total, 7 attack patterns on 73 events during 9 matches were analyzed. The analyzer software output is discussed by the research team, with consciousness of internal validity, allowed by action research method, of this kind of qualitative analysis.

\section{Material}

Two cameras to film the games.

One laptop for analyze end prepare video lessons to show by projector to players during all meetings. Kinovea software was used for select most important part of videos and select the Plate. 


\section{RESULTS}

An evaluation table was constructed by combining, for each single event, the Boolean evaluation of the coach on the compliance of patterns with the event final outcome.

Here are reported

Confrontations of pattern design with Dartfish screenshot of pattern implemented during game; Basic descriptive statistics (occurrence of single patterns on events total, occurrence of "wellperformed pattern" on events total, etc...); Linear regression scatter plot for single patterns;

Confrontation between correlation coefficients of single patterns (referred only to "well-performed" patterns).

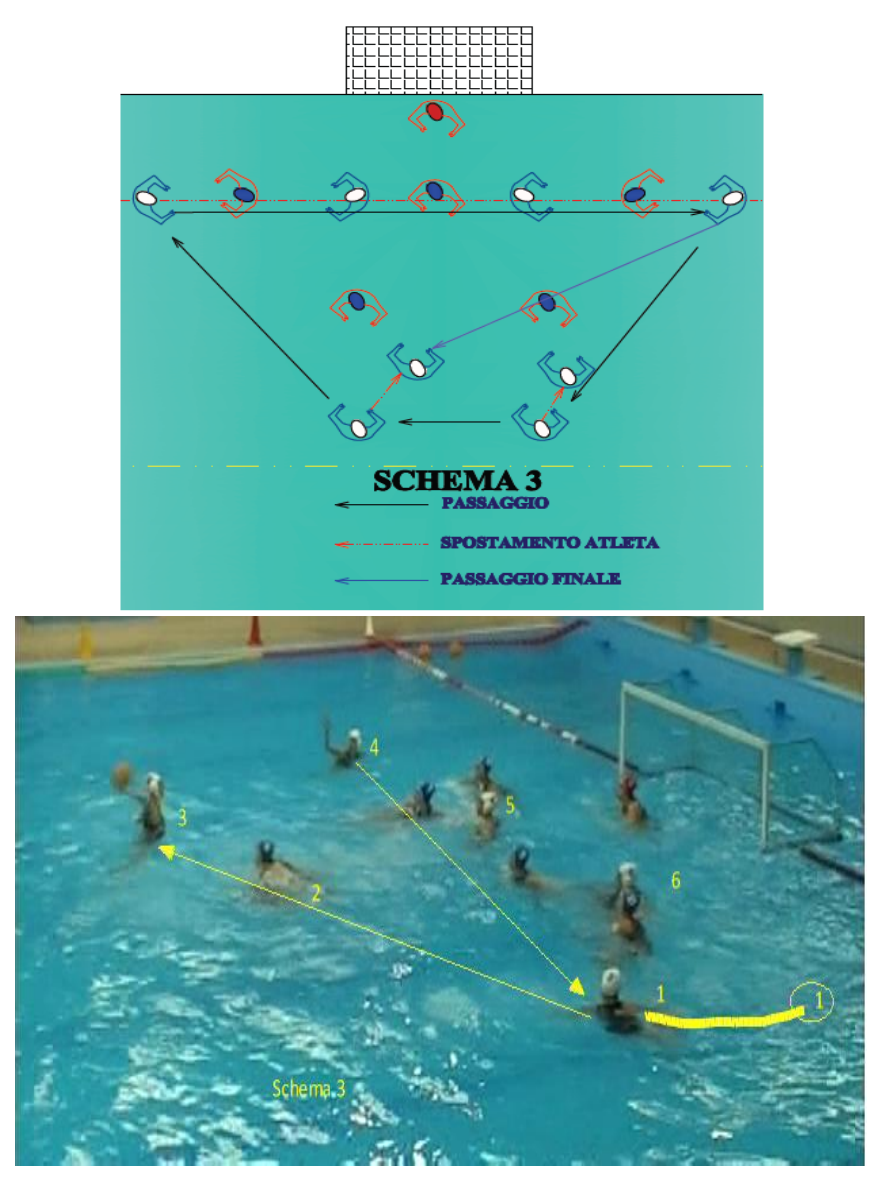

Figure 2. Design of pattern named «schema3»; Plate 2. Pattern «schema3» Screenshot 
Table 1. Table schema 3

\begin{tabular}{ccccc}
\hline Match & $\begin{array}{c}\text { Total } \\
\text { occurrence }\end{array}$ & $\begin{array}{c}\text { Well } \\
\text { performed }\end{array}$ & goals \\
\cline { 2 - 5 } volturno vs orizzonte ct & & 1 & 1 & 1 \\
volturno vs fiorentina fi & & 1 & 1 & 1 \\
volturno vs ortiga sr & & 0 & 0 & 0 \\
volturno vs firenze pn & 1 & 0 & 0 \\
volturno vs padova & 1 & 0 & 0 \\
volturno vs messina & 3 & 1 & 0 \\
volturno vs imperia & 0 & 0 & 0 \\
volturno vs pro recco & 0 & 0 & 0 \\
volturno vs bologna & 1 & 0 & 0 \\
\hline
\end{tabular}

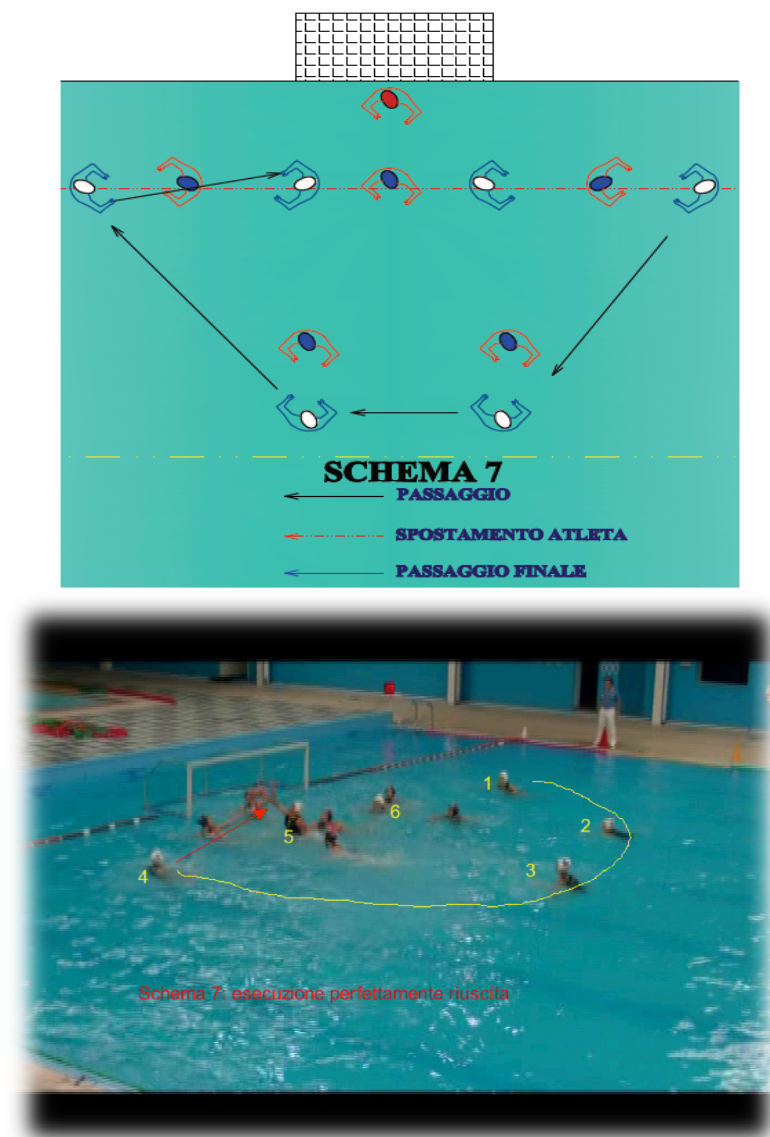

Figure 3. Design of pattern named «schema 7; Plate 3. Pattern «schema7» Screenshot 


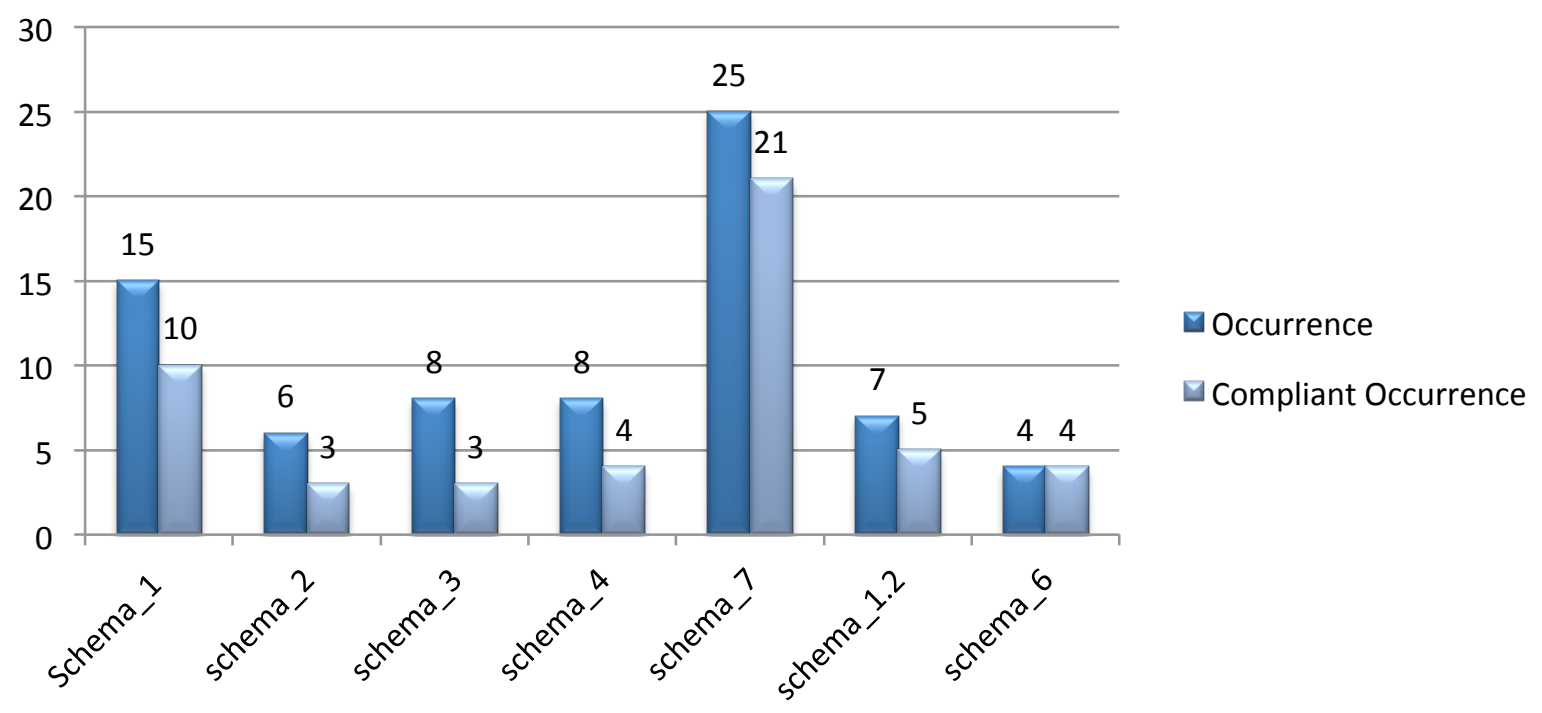

Figure 4. Well-performed patterns occurence

Table 2.

\begin{tabular}{cccc}
\hline ttern & Occurrence & $\begin{array}{c}\text { Compliant } \\
\text { Occurrence }\end{array}$ & Goals \\
\hline Schema_1 & 15 & 10 & 6 \\
schema_2 & 6 & 3 & 3 \\
schema_3 & 8 & 3 & 2 \\
schema_4 & 8 & 4 & 3 \\
schema_7 & 25 & 21 & 13 \\
schema_1.2 & 7 & 5 & 1 \\
schema_6 & 4 & 4 & 2 \\
\hline
\end{tabular}

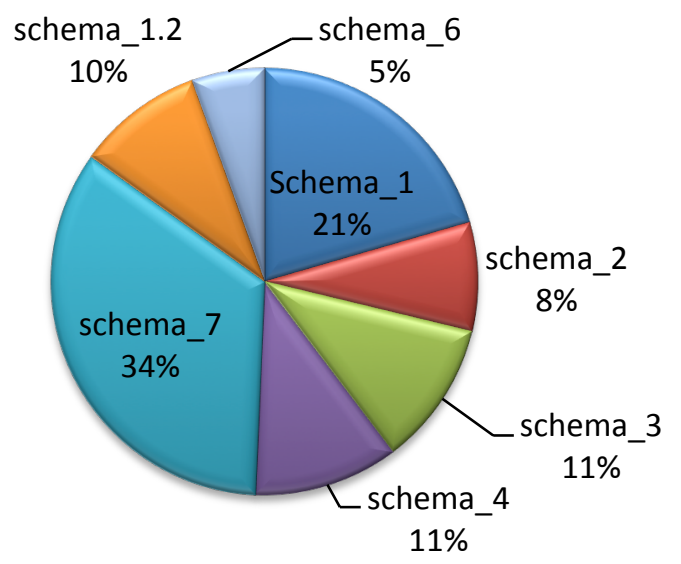

Figure 5. Patterns occurrences -7 attack patterns 
Table 3. Schema correlation

\begin{tabular}{ccc}
\hline Pattern & stderr & $\begin{array}{c}\text { correlation (well performed } \\
\text { patterns /goals }\end{array}$ \\
\hline schema_1 & 0.43678760300431 & 0.76332253361379 \\
schema_2 & 0 & 1 \\
schema_3 & 0.30860669992418 & 0.75592894601845 \\
schema_4 & 0.11624763874382 & 0.98810492932246 \\
schema_7 & 0.54916964736528 & 0.22360679774998 \\
schema_1.2 & 0.34684398780965 & 0.22941573387056 \\
schema_6 & 0.37796447300923 & 0.5976143046672 \\
\hline
\end{tabular}

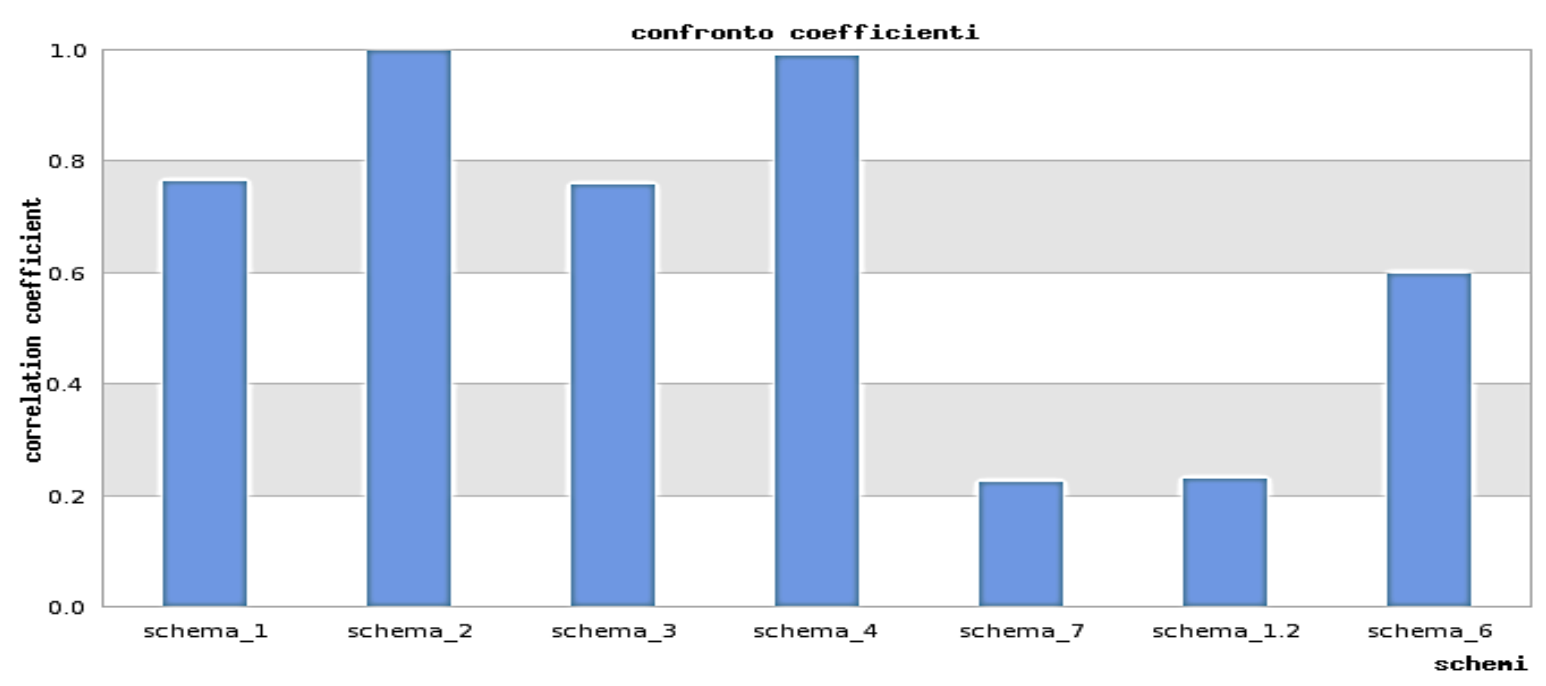

Figure 6. Schema correlation

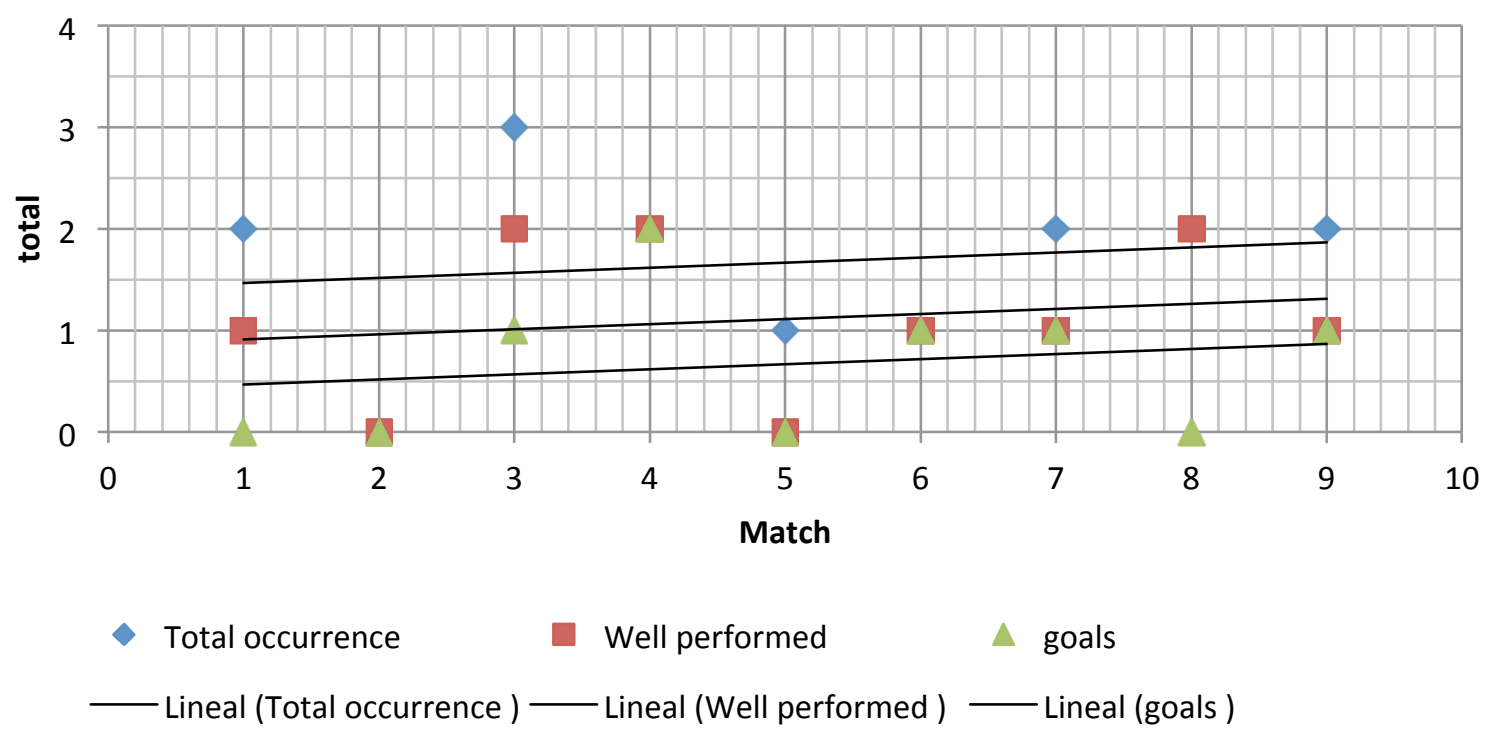

Figure 7. Linear regression. Stder $=0,64-$ corr $=0,53$ 


\section{DISCUSSION}

The results showed a general efficacy of tactical patterns (when they are well performed), but showed significant differences within correlation coefficients of single patterns, knowing that

"the correlation coefficient indicates magnitude or amount of a relationship and the direction of relationship" [Morrow, Jackson].

A more consistent data base is needed, in order to establish direct, evident and general relationship between so calculated coefficient and pattern efficacy, and the research team is conscious of internal validity of this kind of qualitative analysis, which can't extend, without adjustments, to other teams.

\section{CONCLUSIONS}

Although, analysis results represents a tool for the coach, in order to better train team in next season, which were an aim of action research, and showed a general trend on tactical pattern efficacy, which will be deeply investigated in future works.

\section{REFERENCES}

1. Hughes, M. \& Franks, I. (2008). Notational Analysis of Sport: Systems for Better Coaching and Performance in Sport. London: Routledge.

2. Napolitano S., Di Tore, A. \& Raiola G. (2012). Tactical analysis on 9 women water polo methodes during the 2011/12 regular season, op - bn07, 17 th Annual Congres of the ECSS - Bruges - ISBN 978-90902686-8-2.

3. Hughes, M. (2004). Notational analysis - a mathematical perspective. International Journal of Performance Analysis in Sport, 4(2), pp.97-139.

4. Hughes, M. (2007). The essentials of performance analysis: an introduction. United Kingdom: Taylor \& Francis.

5. Raiola, G. \& Di Tore, A. (2011). Applied Regression Analysis: study about the incidence of communication skills on the results of volleyball matches over a full season. Scientific Report Series. Physical Education and Sport, 1/2011, Pitesti: Romania.

6. Schmidt, R. \& Wrisberg, C. (2008). Motor learning and Performance (4th ed.). Champaign, IL: Human Kinetics. 